\title{
Prevalence of cerebrospinal fluid Alzheimer disease-like pattern in atypical dementias
}

\author{
A. Padovani $^{1}$, A. Benussi ${ }^{1}$, F. Ferrari ${ }^{1}$, S. Archetti ${ }^{1,2}$, B. Borroni ${ }^{1 *}$ \\ ${ }^{1}$ Neurology Unit, University of Brescia, Brescia, Italy; ${ }^{\text {Corresponding Author: bborroni@inwind.it }}$ \\ ${ }^{2}$ III Laboratory of Analyses, Brescia Hospital, Brescia, Italy
}

Received 16 October 2012; revised 18 November 2012; accepted 26 November 2012

\begin{abstract}
BACKGROUND: Differential diagnosis between Frontotemporal Dementia (FTD), Corticobasal Syndrome (CBS), Progressive Supranuclear Palsy Syndrome (PSP), FTD with motor neuron disease (FTD-MND) is often challenging, because of the occurrence of atypical cases. Autopsy series have identified Alzheimer Disease (AD) pathology in a consistent percentage of patients with atypical dementias. It has been demonstrated that Cerebrospinal Fluid (CSF) TaulA $\beta_{42}$ dosage is a reliable marker for AD. OBJECTIVE: To evaluate the presence and percentage of CSF $A D$-like patterns (high CSF taulA $\beta_{42}$ ratio) in patients with atypical dementias in order to identify an ongoing $A D$ neurodegenerative process. METHODS: One hundred seventy two consecutive patients fulfilling current clinical criteria for behavioural variant FTD (bvFTD, $n=73$ ), agrammatic variant of Primary Progressive Aphasia (avPPA, $n=19$ ), semantic variant of PPA (svPPA, $n=12)$, FTD-MND $(n=5)$, CBS $(n=42)$, PSP $(n=$ 21) were recruited and underwent $C S F$ analysis. CSF AD-like and non AD (nAD-like) patterns were identified. RESULTS: CSF AD-like pattern was reported in 6 out of 73 cases $(8.2 \%)$ in the bvFTD group, in 3 out of $19(15.8 \%)$ in the avPPA group, and in 7 out of $42(16.7 \%)$ in the CBS group. One out of 12 (8.3\%) of SVPPA had CSF AD-like pattern. None of patients FTD-MND and PSP had CSF AD-like pattern. No differences in demographic characteristics were detected between subgroups in each phenotype. CONCLUSIONS: Our findings convey that the CSF taul $A \beta_{42}$ ratio could be found in a proportion of cases with clinical bvFTD, avPPA and CBD. Detecting an on-going $A D$ pathological process in atypical dementias has several implications for defining distinctive therapeutic approaches, guiding genetic screening and helping in patients' selection in future clinical trials.
\end{abstract}

Keywords: CSF; Alzheimer Disease; Atypical Dementias; Frontotemporal Dementia; Corticobasal Syndrome; Progressive Supranuclear Palsy

\section{INTRODUCTION}

One of the current issues in the spectrum of atypical dementias is the correspondence between phenotypic features and molecular pathology. This issue has crucial implications for understanding disease neurobiology, for clinical diagnosis, and essentially, for development and rational use of disease-modifying therapies [1].

Atypical dementias, as behavioural variant Frontotemporal Dementia (bvFTD), semantic variant of primary Progressive Aphasia (svPPA) and agrammatic variant of PPA (avPPA), FTD with Motor Neuron Disease (FTDMND), Corticobasal Syndrome (CBS), and Progressive Supranuclear Palsy (PSP), are clinically and neuropathologically heterogeneous disorders $[1,2]$.

In many cases, differential diagnosis of these atypical dementias with the most common neurodegenerative disorder, i.e. Alzheimer's disease (AD), is still challenging. The classical model of AD is indeed different, as defined by specific neuropathological characteristics, such as beta amyloid deposition in the brain. Nonetheless, presenile AD patients may depict symptoms overlapping with other neurodegenerative brain disorders $[3,4]$. The phenotypic overlap between neurodegenerative disorders has also been established by neuropathological studies, demonstrating that AD pathology can be found in a significant percentage of patients with alternative clinical diagnosis, as in atypical dementias [5,6]. In several cases, clinical features do not correctly predict the underlying pathology. These findings comply with reports that a substantial minority of patients with $\mathrm{AD}$ present with an atypical phenotype in which amnesia is not the sole initial or predominant clinical feature $[3,4]$.

In the last decade it has been widely accepted that $\mathrm{AD}$ is characterised by a remarkable variation in the concentrations in cerebrospinal fluid (CSF) of $\mathrm{A} \beta_{42}$ and total tau, resulting in reduction of the $\mathrm{CSF} \operatorname{tau} / \mathrm{A} \beta_{42}$ ratio. This 
allows $\mathrm{AD}$ to be distinguished from other neurodegenerative disorders $[7,8]$. Furthermore, neuropathologic data have reported that CSF tau $/ \mathrm{A} \beta_{42}$ ratio has a strong association with $\mathrm{AD}$-related $\mathrm{A} \beta$ senile plaque pathology $[8,9]$. As the biological marker mirrors the pathogenetic mechanism of $\mathrm{AD}$, it can be argued that the CSF tau/ $\mathrm{A} \beta_{42}$ ratio might identify AD not only in the preclinical phase but also in the atypical presentation of the disorder. It could also be hypothesised that $\mathrm{CSF} \operatorname{tau} / \mathrm{A} \beta_{42}$ measurement might be useful in distinguishing cases presenting atypical dementias but with an on-going AD neuropathologycal process.

All the above observations defined the object of this work, aimed at evaluating the presence and percentage of CSF $\mathrm{AD}$-like pattern (i.e., high $\mathrm{CSF}$ tau $/ \mathrm{A} \beta_{42}$ ratio) in consecutive patients with atypical dementias (i.e., bvFTD, svPPA, avPPA, FTD-MND, CBS and PSP).

\section{METHODS}

One hundred seventy two consecutive patients fulfilling current clinical criteria for bvFTD $(\mathrm{n}=73)$, avPPA $(\mathrm{n}=19)$, svPPA $(\mathrm{n}=12)$, CBS (42), PSPS (21), recruited from the Centre for Neurodegenerative Diseases and the Centre for Movement Disorders, University of Brescia, Italy, entered the study. All subjects underwent a somatic and neurological evaluation, routine laboratory examination, received a brain structural study. In all the included subjects, CSF analysis was carried out.

The diagnostic assessment involved a review of full medical history, a semi-structured neurological examination, and a complete mental status evaluation by at least two independent and experienced reviewers. A standardised cognitive and behavioural assessment was carried out, as previously published [7]. Patients were screened for the most common monogenic forms of Granulin (GRN), Microtubule Associated Protein Tau (MAPT), and C9orf72 hexanucleotyde expansion, and genotyped for Apolipoprotein E (ApoE) allelic variations, as already reported [10].

Stringent exclusion criteria were applied as follows: 1) cerebrovascular disorders, previous stroke, hydrocephalus, and intra-cranial mass documented by MRI; 2) a history of traumatic brain injury or another neurological disease; 3) significant medical problems; 4) major depressive disorder, bipolar disorder, schizophrenia, substance abuse disorder. The study was conformed to the Helsinki Declaration and was approved by local Ethic Committee of Brescia, Italy.

\subsection{CSF Analyses}

CSF was obtained during routine diagnostic lumbar puncture according to a standardized protocol, in the outpatient clinic, from 09:30 to 10:30, after informed written consent had been obtained. CSF was collected in sterile polypropylene tubes and gently mixed to avoid gradient effects. Routine chemical measures were determined. The remaining CSF was centrifuged for $3 \mathrm{~min}$ at $3000 \mathrm{rpm}$, and aliquots were stored at $-80^{\circ} \mathrm{C}$ or in liquid nitrogen for subsequent total Tau, phospho-Tau and $\mathrm{A} \beta_{42}$ dosages. CSF concentrations were measured in duplicate by an ELISA test (Innotest hTau antigen kit and Innotest PHOSHO-TAU 181P; Abeta ${ }_{42}$, Innogenetics, Ghent, Belgium). Inter-assay variability was less than $7 \%$. According to our laboratory standards, the cut-off value for total tau was $<400 \mathrm{pg} / \mathrm{ml}$ and for $\mathrm{A} \beta_{42}>400 \mathrm{pg} / \mathrm{ml}$. The $\mathrm{AD}$ pattern was defined by high total tau levels and low $\mathrm{A} \beta_{42}$ levels in CSF and, as previously reported by other authors, with a CSF tau $/ \mathrm{A} \beta_{42}$ ratio $>1$ [11].

\subsection{Statistical Analyses}

Results are expressed as mean \pm standard deviation. Demographic and clinical characteristics between groups were compared with Fisher's exact test or the MannWhitney test, as appropriate. Statistical significance was assumed at $p<0.05$. We calculated the percentage of patients with AD-like CSF pattern for each group. Data analyses were carried out using SPSS 21.0 software.

\section{RESULTS}

Demographic characteristics of the included 172 patients with atypical dementias, according to clinical phenotype, are shown in Table 1. Age at disease onset was comparable in all subgroups but in PSP, who showed older onset. Patients with bvFTD, avPPA, FTDMND and PSP had high percentage of positive family history for dementia ( $\geq 40 \%$ ), as compared to svPPA or CBS.

Frequency of ApoE e4 genotype was higher $(>20 \%)$ in the bvFTD and in the language variants of FTD, i.e. avPPA and svPPA, than in the other phenotypes. According to CSF Abeta and Tau values (see Table 1), as reported in the method section, patients were subdivided into AD-like and no AD-like (nAD) subgroups.

The percentage of patients with CSF AD-like pattern is reported in Figure 1. In bvFTD, 8.2\% (6/73) of patients had CSF AD-like pattern. CSF AD-like pattern was also observed in $15.8 \%(3 / 19)$ of avPPA and in $16.7 \%(7 / 42)$ of CBS patients. In the 12 patients with svPPA, one had CSF AD-like pattern, the percentage being $8.3 \%$. None of patients with the clinical diagnosis FTD-MND and PSP presented CSF AD-like pattern.

No differences in any of the considered demographic and clinical characteristics between patients with ADlike and nAD-like subgroups in ach clinical phenol-type were observed.

When only patients with CSF AD-like pattern were 


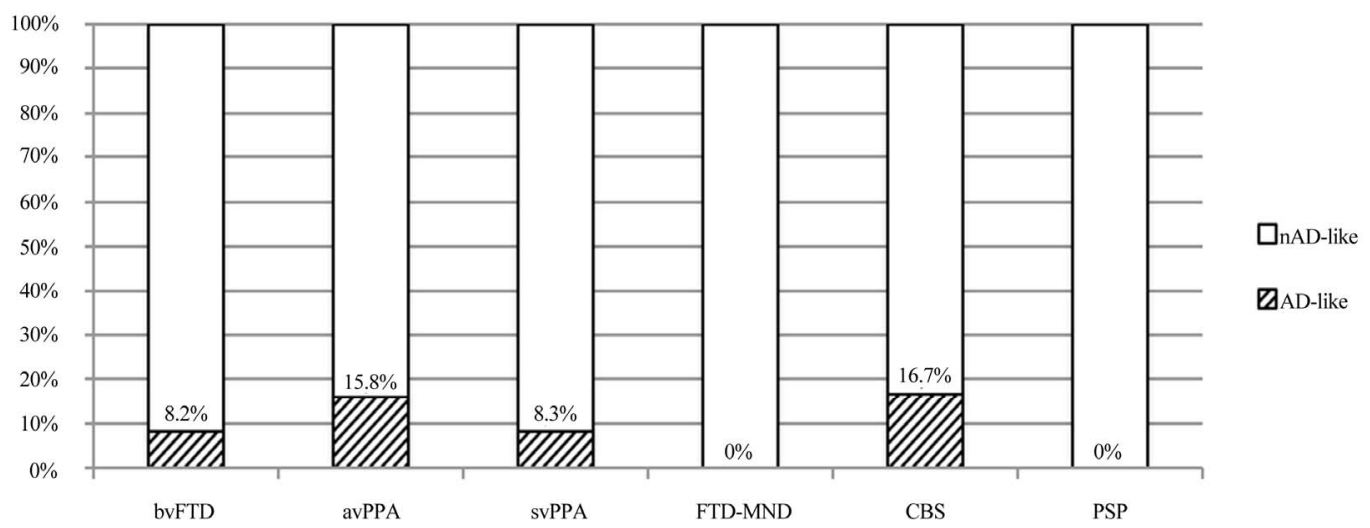

Figure 1. Percentage of CSF AD-like pattern in atypical dementia subgroups. bvFTD: behavioural variant Frontotemporal Dementia; svPPA: semantic variant of Pripmary Progressive Aphasia; avPPA: agrammatic variant of PPA; FTD-MND: FTD with Motor Neuron Disease; CBS: Corticobasal Syndrome; PSP: Progressive Supranuclear Palsy (PSP).

Table 1. Demographic characteristics and CSF analysis of patients with atypical dementias according to clinical phenotype.

\begin{tabular}{|c|c|c|c|c|c|c|}
\hline Variable & bvFTD & svPPA & avPPA & FTD-MND & CBS & PSP \\
\hline $\mathrm{N}$ & 73 & 12 & 19 & 5 & 42 & 21 \\
\hline Age, years & $64.1 \pm 7.0$ & $66 \pm 7.1$ & $63.7 \pm 9.2$ & $66.2 \pm 11.5$ & $64.2 \pm 8.3$ & $71.5 \pm 6.9$ \\
\hline Gender, F\% & 49.3 & 66.7 & 52.6 & 20.0 & 31.0 & 33.3 \\
\hline Education, years & $6.8 \pm 3.0$ & $9.8 \pm 4.8$ & $7.5 \pm 3.5$ & $8.0 \pm 6.3$ & $7.6 \pm 3.6$ & $6.6 \pm 3.4$ \\
\hline Age at onset, years & $61.9 \pm 7.0$ & $63.1 \pm 7.1$ & $61.1 \pm 9.1$ & $63.2 \pm 10.7$ & $60.4 \pm 8.7$ & $67.6 \pm 7.1$ \\
\hline Family history, $\%$ & 44.3 & 16.7 & 42.1 & 75.0 & 26.2 & 40.0 \\
\hline ApoE genotype, $\varepsilon 4 \%$ & 31.9 & 36.4 & 21.0 & 66.6 & 16.6 & 14.2 \\
\hline \multicolumn{7}{|l|}{ CSF analysis } \\
\hline CSF Abeta $_{42}(\mathrm{pg} / \mathrm{ml})$ & $665.8 \pm 305.7$ & $679.8 \pm 429.7$ & $872.6 \pm 451.2$ & $599.1 \pm 304.9$ & $727.4 \pm 399.5$ & $729.9 \pm 357.1$ \\
\hline CSF Tau (pg/ml) & $480.1 \pm 342.1$ & $435.0 \pm 372.6$ & $579.0 \pm 372.0$ & $378.8 \pm 351.7$ & $369.2 \pm 259.0$ & $220.8 \pm 98.9$ \\
\hline CSF P-Tau (pg/ml) & $70.2 \pm 76.7$ & $60.7 \pm 56.0$ & $105.7 \pm 91.5$ & $74.5 \pm 80.2$ & $75.8 \pm 80.5$ & $66.0 \pm 51.2$ \\
\hline CSF Tau/Abeta ratio & $0.95 \pm 0.87$ & $1.14 \pm 1.69$ & $0.93 \pm 0.95$ & $0.65 \pm 0.31$ & $0.96 \pm 1.56$ & $0.36 \pm 0.24$ \\
\hline
\end{tabular}

bvFTD: behavioural variant Frontotemporal Dementia; svPPA: semantic variant of primary Progressive Aphasia; avPPA: agrammatic variant of PPA; FTD-MND: FTD with Motor Neuron Disease; CBS: Corticobasal Syndrome; PSP: Progressive Supranuclear Palsy (PSP); F: Female; ApoE: Apolipoprotein E.

considered, $35 \%$ of patients (6/17) carried ApoE 4 genotype.

\section{DISCUSSION}

In the present work, we investigated the presence and the percentage of CSF AD-like pattern in patients with atypical dementias. We reported that patients with bvFTD, avPPA, and CBS might have two distinct profiles of CSF tau $/ \mathrm{A} \beta_{42}$ ratio, almost $10 \%-15 \%$ of them resembling the biological features of AD. Conversely, none of clinically defined FTD-MND and PSP patients showed CSF AD-like pattern. In regard to svPPA patients, 1 sub- ject out of 12 had high Tau and low Abeta 42 levels. No differences in demographic characteristics were detected between subgroups.

Up to now, no biomarker has been identified to increase the diagnostic accuracy of FTD spectrum. This could be rationalized by both the neuropathological heterogeneity and the substantial variation in neurodegenerative severity between early and late stages of disease [12]. Indeed, different neuropathological profiles have been described in FTD. BvFTD has been observed in association with a number of pathological hallmarks (i.e. tau, FTLD-TDP type A, FUS), with the exception of FTLDTDP type B and C pathology [1]. svPPA has been mostly 
associated with FTLD-TDP type C [10] pathology and only rarely with FTLD-tau or AD pathology $[4,13,14]$. avPPA has been commonly associated with FTLD-tau $[13,15,16]$, AD and, to a lesser extent, FTLD-TDP pathology [4,14]. FTD-MND has been associated with FTLD-TDP type B pathology $[17,18]$. Accordingly, in FTD spectrum in most cases CSF tau and Abeta levels have resulted within normal range, but in some cases a slight increase or even decrease has been also found [19].

In CBS, the findings on CSF Tau and Abeta $a_{42}$ dosages are highly heterogeneous. CSF Tau levels were reported higher than control subjects in two studies [20,21], whereas three other studies showed no significant differences between CBS and patients affected by other neurodegenerative disorders with distinct tau related pathology $[22,23]$. Nevertheless, the high variability of all the above studies highlights the presence of different associated neuropathological features. Corticobasal Degeneration (CBD) has a distinct neuropathological profile characterized by extensive neuronal and glial taupositive inclusions; however, there is growing evidence that these patients may have alternative pathologies $[24,25]$ including AD, PSP and FTD which lead to the use of the Corticobasal Syndrome (CBS) label [26,27].

If in bvFTD, the language-related dementias and CBS indicate that an ongoing $\mathrm{AD}$ neuropathological process may occur, in PSP and FTD-MND cases CSF analysis suggests a unique relationship between clinical and neuropathological diagnosis. In PSP, a degenerative tauopathy with $4 \mathrm{R}$ tau accumulation [28], CSF tau levels were reported within the normal range and comparable to those obtained in control subjects [29]. According to this, neuropathological findings suggest that clinical diagnosis of PSP is highly suggestive of PSP pathology. In the same view, in FTD-MND no CSF AD-like pattern was observed.

It has been extensively demonstrated that AD is associated with a specific pattern of CSF abnormalities (high tau and low $\mathrm{A} \beta_{42}$ levels) compared with other neurodegenerative disorders [8]. To date, $\mathrm{CSF}$ tau $/ \mathrm{A} \beta_{42}$ ratio has been widely suggested as a supportive criterion for preclinical AD diagnosis [30].

In this study we have evaluated the hypothesis that different CSF patterns, AD-like and nAD-like, might define distinct phenotypes in FTD, CBS and PSP. In fact, we might argue that CBS and FTD patients with CSF ADlike pattern are instead cases with atypical and focal $A D$ pathology $[4,25,31]$. The percentage of patients reclassified by CSF analysis in AD-like is in line with several pathological series [32]. On the other hand, we cannot rule out that CSF AD-like variations may co-occur in patients with atypical dementias [11], as recently highlighted by a recent study, in which almost $25 \%$ of the cases presented with multiple neurodegenerative patho- logies [33]. In both cases, patients with distinctive $\mathrm{AD}$ pathological features and alternative clinical diagnosis should be treated with a different therapeutical viewpoint.

The CSF tau/ $\mathrm{A} \beta_{42}$ ratio has widely been proven as a highly specific hallmark for AD. In contrast, no precise CSF pattern has been identified in patients with atypical dementias. Nevertheless, we think that CSF analyses should be undertaken in patients with clinical CBS or FTLD diagnosis, as an exclusion criterion. In clinically defined PSP and FTD-MND cases, an ongoing AD pathological process is less likely.

We acknowledge that this work has some limitations. Firstly, the neuropathological confirmation and the combined use of amyloid $\beta$ tracers would be mandatory to definitely clarify the present findings [34]. Secondly a more attentive neuropsychological analysis might be of help for further differentiating AD-like and nAD-like subgroups. Thirdly, CSF analyses were not performed in patients with Logopenic Progressive Aphasia (LPA), a recently defined subtype of PPA, predominantly associated with AD pathology. Finally, follow-up might be compelling in distinguishing different courses of disease progression in the two subgroups.

In conclusion, these findings convey that the CSF $\operatorname{tau} / \mathrm{A} \beta_{42}$ ratio could be a possible path in discerning $\mathrm{AD}$ patients with atypical presentation from patients with atypical dementias. If these findings will be confirmed by additional studies, patients with and AD-like CSF pattern should be treated with a different analeptic approach, and current pharmacological treatment for $\mathrm{AD}$ should be evaluated.

\section{REFERENCES}

[1] Rohrer, J.D., Lashley, T., Schott, J.M., et al. (2011) Clinical and neuroanatomical signatures of tissue pathology in frontotemporal lobar degeneration. Brain, 134, 2565-2581. doi:10.1093/brain/awr198

[2] Rascovsky, K., Hodges, J.R., Knopman, D., et al. (2011) Sensitivity of revised diagnostic criteria for the behavioural variant of frontotemporal dementia. Brain, 134, 2456-2477. doi:10.1093/brain/awr179

[3] Lopez, O.L., Becker, J.T., Klunk, W., et al. (2000) Research evaluation and diagnosis of probable Alzheimer's disease over the last two decades: I. Neurology, 55, 18541862. doi:10.1212/WNL.55.12.1854

[4] Cummings, J.L. (2000) Cognitive and behavioral heterogeneity in Alzheimer's disease: Seeking the neurobiological basis. Neurobiology of Aging, 21, 845-861. doi:10.1016/S0197-4580(00)00183-4

[5] Kertesz, A., McMonagle, P., Blair, M., et al. (2005) The evolution and pathology of frontotemporal dementia. Brain, 128, 1996-2005. doi:10.1093/brain/awh598

[6] Forman, M.S., Farmer, J., Johnson, J.K., et al. (2006) 
Frontotemporal dementia: Clinicopathological correlations. Annals of Neurology, 59, 952-962.

doi:10.1002/ana.20873

[7] Visser, P.J., Verhey, F., Knol, D.L., et al. (2009) Prevalence and prognostic value of CSF markers of Alzheimer's disease pathology in patients with subjective cognitive impairment or mild cognitive impairment in the DESCRIPA study: A prospective cohort study. The Lancet Neurology, 8, 619-627.

doi:10.1016/S1474-4422(09)70139-5

[8] Mattsson, N., Zetterberg, H., Hansson, O., et al. (2009) CSF biomarkers and incipient Alzheimer disease in patients with mild cognitive impairment. The Journal of the American Medical Association, 302, 385-393. doi:10.1001/jama.2009.1064

[9] Tapiola, T., Alafuzoff, I., Herukka, S.-K., et al. (2009) Cerebrospinal fluid \{beta\}-amyloid 42 and tau proteins as biomarkers of Alzheimer-type pathologic changes in the brain. Archives of Neurology, 66, 382-389. doi:10.1001/archneurol.2008.596

[10] Premi, E., Garibotto, V., Alberici, A., et al. (2012) Nature versus nurture in frontotemporal lobar degeneration: the interaction of genetic background and education on brain damage. Dementia and Geriatric Cognitive Disorders, 33, 372-378. doi: $10.1159 / 000339366$

[11] Bian, H., Van Swieten, J.C., Leight, S., et al. (2008) CSF biomarkers in frontotemporal lobar degeneration with known pathology. Neurology, 70, 1827-1835.

[12] Seelaar, H., Rohrer, J.D., Pijnenburg, Y.A.L., et al. (2011) Clinical, genetic and pathological heterogeneity of frontotemporal dementia: A review. Journal of Neurology, Neurosurgery \& Psychiatry, 82, 476-486. doi:10.1136/jnnp.2010.212225

[13] Davies, R.R., Hodges, J.R., Kril, J.J., et al. (2005) The pathological basis of semantic dementia. Brain, 128, 19841995. doi:10.1093/brain/awh582

[14] Grossman, M. (2010) Primary progressive aphasia: Clinicopathological correlations. Nature Reviews. Neurology, 6, 88-97. doi:10.1038/nrneurol.2009.216

[15] Mesulam, M., Wicklund, A., Johnson, N., et al. (2008) Alzheimer and frontotemporal pathology in subsets of primary progressive aphasia. Annals of Neurology, 63, 709719. doi:10.1002/ana. 21388

[16] Deramecourt, V., Lebert, F., Debachy, B., et al. (2010) Prediction of pathology in primary progressive language and speech disorders. Neurology, 74, 42-49. doi:10.1212/WNL.0b013e3181c7198e

[17] Cairns, N.J., Bigio, E.H., Mackenzie, I.R., et al. (2007) Neuropathologic diagnostic and nosologic criteria for frontotemporal lobar degeneration: Consensus of the Consortium for Frontotemporal Lobar Degeneration. Acta Neuropathologica, 114, 5-22. doi:10.1007/s00401-007-0237-2

[18] Sampathu, D.M., Neumann, M., Kwong, L.K., et al. (2006) Pathological heterogeneity of frontotemporal lobar degeneration with ubiquitin-positive inclusions delineated by ubiquitin immunohistochemistry and novel monoclonal antibodies. The American Journal of Pathology, 169,

\section{3-1352. doi:10.2353/ajpath.2006.060438}

[19] Verwey, N.A., Kester, M.I., van der Flier, W.M., et al. (2010) Additional value of CSF amyloid-beta 40 levels in the differentiation between FTLD and control subjects. Journal of Alzheimer's Disease, 20, 445-452.

[20] Urakami, K., Wada, K., Arai, H., et al. (2001) Diagnostic significance of tau protein in cerebrospinal fluid from patients with corticobasal degeneration or progressive supranuclear palsy. Journal of the Neurological Sciences, 183, 95-98. doi:10.1016/S0022-510X(00)00480-9

[21] Paraskevas, G.P. (2005) The diagnostic value of cerebrospinal fluid tau protein in sementing and nondementing neuropsychiatric disorders. Journal of Geriatric Psychiatry and Neurology, 18, 163-173. doi: $10.1177 / 0891988705277549$

[22] Arai, H., Morikawa, Y., Higuchi, M., et al. (1997) Cerebrospinal fluid tau levels in neurodegenerative diseases with distinct tau-related pathology. Biochemical and Biophysical Research Communications, 236, 262-264. doi:10.1006/bbrc.1997.6908

[23] Noguchi, M., Yoshita, M., Matsumoto, Y., et al. (2005) Decreased beta-amyloid peptide42 in cerebrospinal fluid of patients with progressive supranuclear palsy and corticobasal degeneration. Journal of the Neurological Sciences, 237, 61-65. doi:10.1016/j.jns.2005.05.015

[24] Sha, S., Hou, C., Viskontas, I.V., et al. (2006) Are frontotemporal lobar degeneration, progressive supranuclear palsy and corticobasal degeneration distinct diseases? $\mathrm{Na}$ ture Clinical Practice. Neurology, 2, 658-665. doi:10.1038/ncpneuro0357

[25] Chand, P., Grafman, J., Dickson, D., et al. (2006) Alzheimer's disease presenting as corticobasal syndrome. Movement Disorders, 21, 2018-2022. doi: $10.1002 / \mathrm{mds} .21055$

[26] Boeve, B.F., Lang, A.E. and Litvan, I. (2003) Corticobasal degeneration and its relationship to progressive supranuclear palsy and frontotemporal dementia. Annals of Neurology, 54, S15-S19. doi:10.1002/ana.10570

[27] Lang, A.E. (2003) Corticobasal degeneration: Selected developments. Movement Disorders, 18, S51-S56. doi: $10.1002 / \mathrm{mds} .10563$

[28] Dickson, D.W. (2012) Parkinson's disease and parkinsonism: Neuropathology. Cold Spring Harbor Perspectives in Medicine, 2, a009258.

[29] Borroni, B., Malinverno, M., Gardoni,F., et al. (2008) Tau forms in CSF as a reliable biomarker for progressive supranuclear palsy. Neurology, 71, 1796-1803. doi:10.1212/01.wnl.0000335941.68602.39

[30] Dubois, B., Feldman, H.H., Jacova, C., et al. (2007) Research criteria for the diagnosis of Alzheimer's disease: Revising the NINCDS-ADRDA criteria. The Lancet Neurology, 6, 734-746. doi:10.1016/S1474-4422(07)70178-3

[31] Hu, W.T., Rippon, G.W., Boeve, B.F., et al. (2009) Alzheimer's disease and corticobasal degeneration presenting as corticobasal syndrome. Movement Disorders, 24, 13751379. doi: $10.1002 / \mathrm{mds} .22574$

[32] Grossman, M., Libon, D.J., Forman, M.S., et al. (2007) Distinct antemortem profiles in patients with pathologi- 
cally defined frontotemporal dementia. Archives of $\mathrm{Neu}$ rology, 64, 1601-1609. doi:10.1001/archneur.64.11.1601

[33] Jellinger, K.A. and Attems, J. (2010) Prevalence of dementia disorders in the oldest-old: An autopsy study. Acta Neuropathology, 119, 421-433.

$$
\text { doi:10.1007/s00401-010-0654-5 }
$$

[34] Cohen, A.D., Rabinovici, G.D., Mathis, C.A., et al. (2012) Using pittsburgh compound B for in vivo PET imaging of fibrillar amyloid-beta. Advances in Pharmacology, 64, 27-81. doi:10.1016/B978-0-12-394816-8.00002-7 